\title{
Child behaviour after anaesthesia: associated risk factors
}

\author{
M Karling (mats.karling@vll.se), ${ }_{1}$ H Stenlund, ${ }^{2}$ B Hägglöf ${ }^{3}$ \\ 1.Division of Anaesthesia, University of Umeå, Sweden \\ 2.Department of Public Health and Clinical Medicine, University of Umeå, Sweden \\ 3.Epidemiology and Child and Adolescent Psychiatry, University of Umeå, Sweden
}

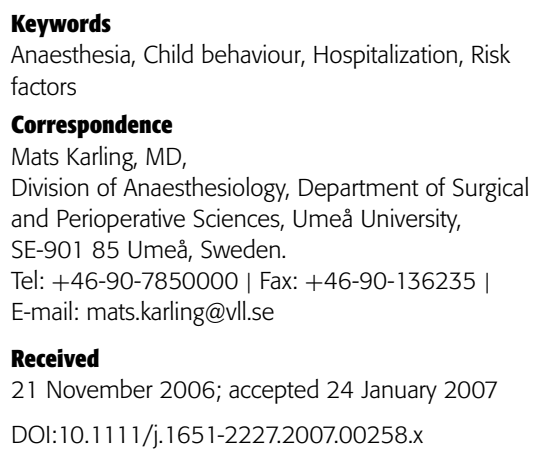

\begin{abstract}
Aim: To identify hospital care factors which are associated with problematic behaviours in children after hospitalization.

Method: A cohort of 340 children ages 2-13 was studied in connection with elective procedures which included anaesthesia. Data collected: sociodemograhic, type of procedure, anaesthesia induction technique and premedication. Staff and parents assessed child anxiety at induction of anaesthesia, pain, anxiety and nausea in recovery room and hospital ward. Parents assessed their child's pain and nausea and the behaviour measured with the Post Hospital Behavioural Questionnaire two weeks after hospitalization.

Results: One-third (34.4\%) of the children developed at least one problematic behaviour, measured by the PHBQ subscales. Multiple logistic regression identified the following risk factors: age $<5$, pain at home but not at hospital, nausea, child anxiety at anaesthesia induction, postoperative nausea, postoperative distress, previous hospitalizations, living in a one adult family and having some previous problematic behaviours. Moderate-to-severe pain at home, but not at hospital, was associated with the greatest risk (OR $6.39 \mathrm{Cl}$ : 3.53-11.6). Previous anaesthesia, midazolam use in premedication and living in rural areas seemed to be protective factors.

Conclusion: Pain at home but not in hospital is a strong risk factor for the onset or worsening of problematic

behaviour after childhood hospitalization, which included anaesthesia. Proactive interventions are suggested to prevent this by improving pain treatment at home.
\end{abstract}

\section{INTRODUCTION}

Hospitalization of a child is associated with many threatening events for both child and parents. This process often involves pain, separation from parents, strange and unknown environments, fear of bodily harm (1). It is well known that children may react to these events with negative behavioural problems (2). Behaviours like nightmares, sleep and eating problems, separation anxiety and regression have been described (3). The Post Hospital Behaviour Questionnaire (PHBQ) (4) is the most widely used questionnaire to measure behavioural change after hospitalization of children. This instrument has recently been translated into Swedish and its psychometric properties (5) and association with sociodemographic data has been evaluated. Its association with the Child Behaviour Checklist (CBCL) (6-8) further strengthens its use for assessing child behaviour.

There have been investigations of hospitalization factors that represent risk for problematic behavioural outcomes (9-11). The following risk factors and prophylactic interventions have been described: anxiety at anaesthesia induction $(9,12-14)$, parental presence at anaesthesia induction $(15,16)$, previous hospitalizations especially with upsetting experiences $(9,10,17,18)$, anxiolytic premedication (19-22), type of anaesthesia induction $(14,23,24)$, day care versus inpatient treatment $(17,18,25-27)$ and different types of prepa- ration programs $(1,11,28,29)$. Several reviews in the subject have been published (3,30-32).

There have been no studies to date in Sweden to investigate the effect of hospitalization, surgery and anaesthesia on subsequent behavioural changes, and few studies $(10,33)$ have assessed the impact of pain at home on behaviour. The purpose of this study was to assess the frequency of behavioural changes in children after hospitalization including anaesthesia, and to find factors in the hospital care that might be associated with these changes two weeks after hospitalization.

\section{STUDY DESIGN}

A cohort of children, ages 2-13 (mean $7.0 \pm 2.9$ ), who were scheduled for an elective procedure that included anaesthesia, either because of surgery or for diagnostic purposes, were followed from 2 weeks before hospitalization, during hospital care and for 2 weeks after leaving the hospital. The children were recruited consecutively from hospital anesthesia schedules. Children with oncological disease, mental retardation or with parents who could not speak or understand Swedish were excluded. The Regional Ethics Committees at Umeå and Gothenburg Universities approved the study.

Two weeks before admittance, the parents were asked to complete a questionnaire concerning demographic data, 
number of previous hospitalizations and anaesthesias. At the same time parents were asked to complete the Swedish version of the Child Behaviour Checklist (CBCL/23 (7) or CBCL/4-18 (6)).

A short written form, including information about the hospital procedure, blood tests, premedication and anaesthesia, was sent to the parents at the same time (Figure S1).

During hospitalization the responsible nurse filled in forms concerning the type of procedure, pain, anxiety, nausea. If premedication was used, the drug was noted and the type of anaesthesia induction: mask, intravenous or rectal. A parent was present at anaesthesia induction and the nurse assistant recorded anxiety of child and parent at the same time. The personal contact with the parent and child was assessed: easy good contact or parent difficult to reach, shielded.

In the recovery room and hospital ward pain, nausea and anxiety was recorded every third hour until these problems was controlled or patient returned home. Parents used a form where they recorded pain, anxiety and distress of their child at anaesthesia induction, in the recovery room and in case of an inpatient care, at the ward. When leaving hospital, the child, if possible, and parent were separately asked about the pain during hospitalization and these responses were recorded as follows: usual pain, worst pain and pain now.

Two weeks after returning home, a letter was sent to the parents containing the PHBQ-form and questions about pain, usual and worst, and nausea at home. They were also asked about the duration of pain in days. If not answered within one week, a reminder was sent.

\section{PATIENTS AND METHODS}

Data were collected during 2002 and 2003. All children except 14 were treated in the University Hospital of Northern Sweden, Umeå. The rest were from Queen Silvia Child and Youth Hospital in Gothenburg, Sweden. This general database has been described in earlier publications $(5,8)$. Number of participants and dropouts are illustrated in Figure S1. There were no difference in age or gender between dropouts and participants. Mean age for dropouts were 6.78 and for the participants $6.97(\mathrm{p}=0.45, t$-test). There were $59 \%$ boys, $41 \%$ girls among the participants and $65 \%$ boys, $35 \%$ girls among the dropouts $(\mathrm{p}=0.18$, Chi-square test).

\section{The CBCL}

The CBCL consists of two parts: social competence scale and behaviour/emotional problems. In this study only the items from the behavioural/emotional portion was used. This included 100 items for children between 2 and 4 years of age and 113 for children from 4 years of age and older. The parents were asked to answer the items with a three-point scale: 0 - do not agree; 1 - agrees somewhat or sometimes; 2 - fully agree. The CBCL behavioural/emotional portion is divided into two broadband dimensions: Internalizing and Externalizing and those are divided in subscales. In a former study, it was found that children with higher CBCL scores before hospitalization, had higher PHBQ scores (8). In order to confirm this finding the total score was dichotomized with a cut-off point using the mean of the medians of the two populations. The value used was 22 for the younger population and 17.5 for the older.

\section{The PHBQ}

The PHBQ was chosen as the primary outcome variable, it consists of 25 items concerning child behaviour (5). The original PHBQ existed in two forms: an absolute form where the parents assess their child before and after hospitalization and a relative form where they are asked to estimate any change in behaviour compared to the time before hospitalization. In this study the relative form was used. Five alternatives for each behaviour item are provided: $1=$ much less; $2=$ less; $3=$ unchanged; $4=$ more; $5=$ much more. The total score was computed by adding the individual item scores. Each total score could be dived into five subscales: General anxiety withdrawal, Eating disturbances, Separation anxiety, Regression-aggression and Sleep anxiety (5). When analysing the relation to any potential risk factor, the PHBQ and its subscales were dichotomized: no change or less problematic behaviours, and more problematic behaviours.

\section{Anxiety and distress}

Parent and child anxiety at anaesthesia induction was assessed by both parent and staff using a $100-\mathrm{mm}$ visual analogue scale (VAS). Staff assessed the emotional contact with parent and child using a 100-mm VAS scale, where 100 $\mathrm{mm}$ was indicating no contact. Parent and staff assessed postoperative distress: staff used a three-grade Likert scale: $0=$ No distress; $1=$ some distress; $2=$ much distress. In the data analysis 1 and 2 were grouped together. Parent assessed distress at two different times: in the recovery room and at the ward. A visual analogue scale of $100 \mathrm{~mm}$ was used. The mean of those values were calculated, if any of those two measurements were missing (e.g. day cases) the other was used.

Parent assessed how well prepared/informed child and parent were regarding the procedure. The VAS scale was used and $100 \mathrm{~mm}$ indicated poorly prepared.

\section{Pain and nausea}

Pain was measured by self assessment using a six-graded faces scale (34) or, for children younger than 4-5 years of age, by observation and/or interrogation to child or parent using three graded scale (no pain, some pain, much pain). Parent assessed the pain of their child using a VAS scale. Nausea was assessed by a three-graded Likert scale: no nausea, some nausea, much nausea.

Hospital pain data were collected from assessments done by the child, staff and parents and were transformed in one number. When self assessed data were missing primarily parent data and secondarily staff data were used in order to reduce the number of missing data. Nausea at home were assessed by a 100-mm VAS scale 


\section{Statistics}

Logistic regression was used to establish the association between risk factors and outcome. Primarily univariate analysis was performed and any risk factor that showed significant correlation to the total PHBQ score or any of its subscales were entered in the multiple regression model. Comparisons between groups were done by Mann-Whitney test and Chisquare for frequencies. All statistical analysis was made by using SPSS 13.0.

\section{RESULTS}

Data were collected before (Table S1) during (Tables S2 and S3) and after hospitalization (Table S4). Type of procedures and length of stay are presented in Table S5.

\section{Child and parent emotions and reactions at anaesthesia induction}

Child anxiety was assessed by both parents and staff. The assessments were highly correlated $(\mathrm{r}=0.6, \mathrm{p}<0.001$ Pearson correlation). Staff evaluated emotional contact with child correlated well with anxiety $(r=0.7, \mathrm{p}<0.001)$. Staff evaluation of parent anxiety and contact was highly correlated $(\mathrm{r}=$ $0.9, \mathrm{p}<0.001$ ), but was only weakly correlated to the emotions rated by the parent $(\mathrm{r}=0.3, \mathrm{p}<0.001$ for anxiety and $\mathrm{r}=0.2, \mathrm{p}<0.001$ for contact). Parent assessments of their own and child anxiety was weakly associated $(\mathrm{r}=0.2, \mathrm{p}<$ $0.001)$. Thus, only parent rating of child anxiety and own anxieties were used for further calculations (Table S2). When missing data from parent assessment $(23 ; 6.7 \%$ for child anxiety, 24; 7.0\% for parent anxiety) the assessment from staff was used and thus reducing dropout to 10 (2.9\%) for child anxiety and $13(3.8 \%)$ for parent anxiety. Moderateto-severe anxiety at anaesthesia induction was found in 15\% of the children and $10 \%$ of the parents assessed themselves as very worried.

The parent assessment of how well they were prepared for or informed about the procedure correlated to the anxiety of their own $(\mathrm{r}=0.3, \mathrm{p}<0.001)$ and their assessment of child anxiety $(r=0.4, p<0.001)$ but not to staff assessment of child anxiety.

\section{Pain}

At hospital, 29\% assessed their usual pain as moderate-tosevere pain and at home $28 \%$. Tonsillectomy was associated with significant longer pain duration at home compared with all other groups ( 7.25 vs. 2.16 days mean, $\mathrm{p}<0.001$ ). Children after tonsillectomy had significantly more usual pain at home than all other groups except adenoidectomy, orchidopexy and strabismus surgery ( $52 \%$ vs. $17 \%, \mathrm{p}<0.001)$. Tonsillectomy was associated with significantly more moderate-tosevere worst pain at home than all other except strabismus surgery $(73 \%$ vs. $25 \%, \mathrm{p}<0.001)$.

\section{Nausea}

Nausea at hospital affected $25 \%$ of the children while $15 \%$ had moderate-to-severe nausea at home. Tonsillectomy was significantly associated with more nausea at hospital than other procedures. (37.5\% vs. $17.8 \%, \mathrm{p}<0.001)$. At home there was no difference between groups (12.5\% vs. $15.3 \%$, NS).

\section{PHBQ}

When analysing the total score, 273 (71.5\%) had unchanged or improved PHBQ score indicating no problematic behaviour outcome on average, while 97 (28.5\%) had scores indicating deterioration in behaviour (Figure S2). The frequencies for negative outcome in the subscales were $10 \%$ for General anxiety withdrawal and between $13.5 \%$ and $15 \%$ for the other subscales. When looking at individual items, 117 $(34.4 \%)$ patients had new onset of negative behaviours and $93(27.3 \%)$ had new positive behaviours onset. The mean number of negative behavioural changes was 1.17 per child.

The influences of different factors collected during the procedure were analysed using multiple logistic regression, calculating odds ratio and confidence interval. Worst pain at home, moderate-to-severe, living in a one adult family and child anxiety at anaesthesia induction, were most strongly associated to problematic behaviour according to the total PHBQ score. Living in an urban area (>10,000 inhabitants), postoperative distress and nausea were also significantly related to a problematic outcome, while being older than 5 years was a protecting factor (Figure 1).

Having more than one sibling was related to increased separation anxiety. Repeated $(>1)$ previous hospitalizations seems to be a risk to develop increased general anxiety/withdrawal while previous anaesthesias reduced the risk. Children having more problematic behaviour prior to hospitalization measured by the CBCL developed more regressive/aggressive behaviour and had more problems in connection with sleep. Inpatient care was associated with more separation anxiety, while stay more than one night at hospital increased the risk for anxiety/withdrawal. Tonsillectomy was connected to later eating disturbances and increased regression/aggression. Moderate-to-severe pain in the recovery room and nausea at home was associated with increased risk of eating disturbances. Premedication with midazolam was protective against regressive-aggressive behaviour and children from families with mothers with lower education seem to have less general anxiety/withdrawal behaviour than those from families with university educated mothers.

\section{DISCUSSION}

In this study the influence on behaviour by hospitalization including a diagnostic or surgical procedure requiring anaesthesia was investigated. We have previously analysed the impact of sociodemographic (8) factors, and this study is a further step in an attempt at comprehensive analysis of relevant risk factors in the same population. In addition to these data, it appears that pain, especially at home after hospitalization, is a major risk factor. Other risk factors were child anxiety at anaesthesia induction, postoperative distress and nausea. Tonsillectomy was associated with more pain and negative behavioural outcome. Day care and midazolam in premedication as well as previous anaesthesia experience had some protective effect. 


\section{Total PHBQ score}

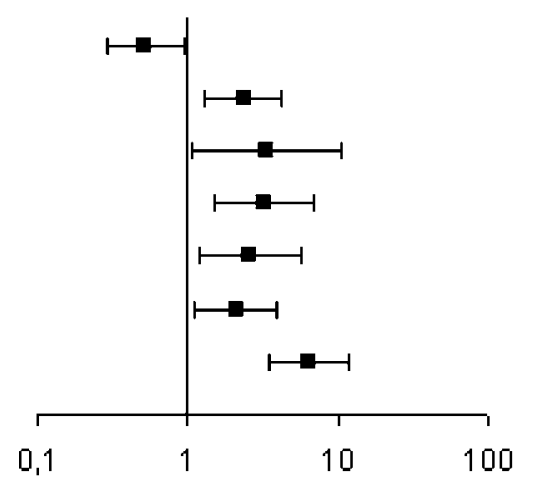

Age more than 5 years

Domicile in urban area

Only one adult in family

Child anxious at anaesthesia start

Postoperative distress

Postoperative nausea

Worst pain at home moderate to severe

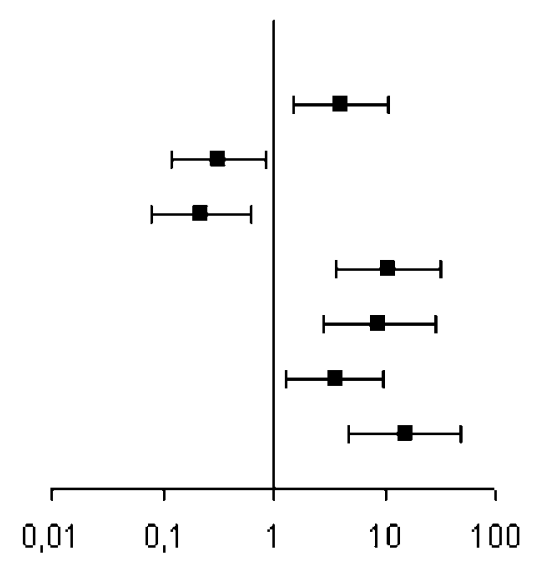

\section{General Anxiety withdrawal}

More than 1 previous hospitalization

Mother education lower than university

Previous anaesthesias vs. None

More than 1 night at hospital

Child anxious at anaesthesia start

Postoperative nausea

Worst pain at home moderate to severe

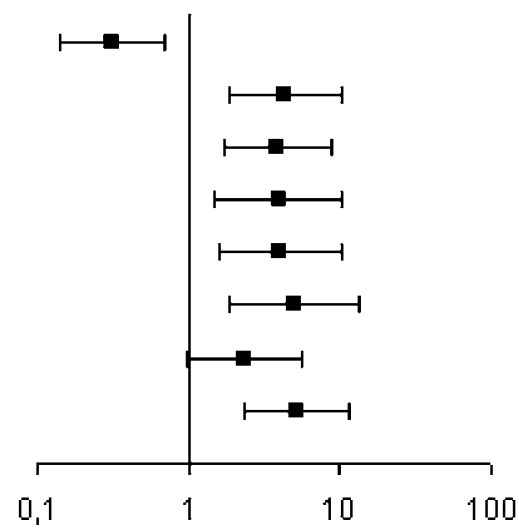

\section{Separation anxiety}

Age more than 5 years

Domicile in urban area

More than 2 children in family

Inpatient care vs. Day care

Child anxious at anaesthesia start

Postoperative distress

Postoperative nausea

Usual pain at home moderate to severe

0,1

10

100

Figure 1 Multiple logistic regressions. Odds Ratio (- $)$ and 95\% confidence interval, only significant outcomes are shown.

Data concerning sociodemography, previous experiences and behavioural problems

The findings in the previous investigation was that age, one adult family, and living in an urban area were risk factors for problematic PHBQ total score outcome (8). When adding information about the hospital procedure in the present study those factors were still associated with problematic outcome. There were some minor differences in that one 

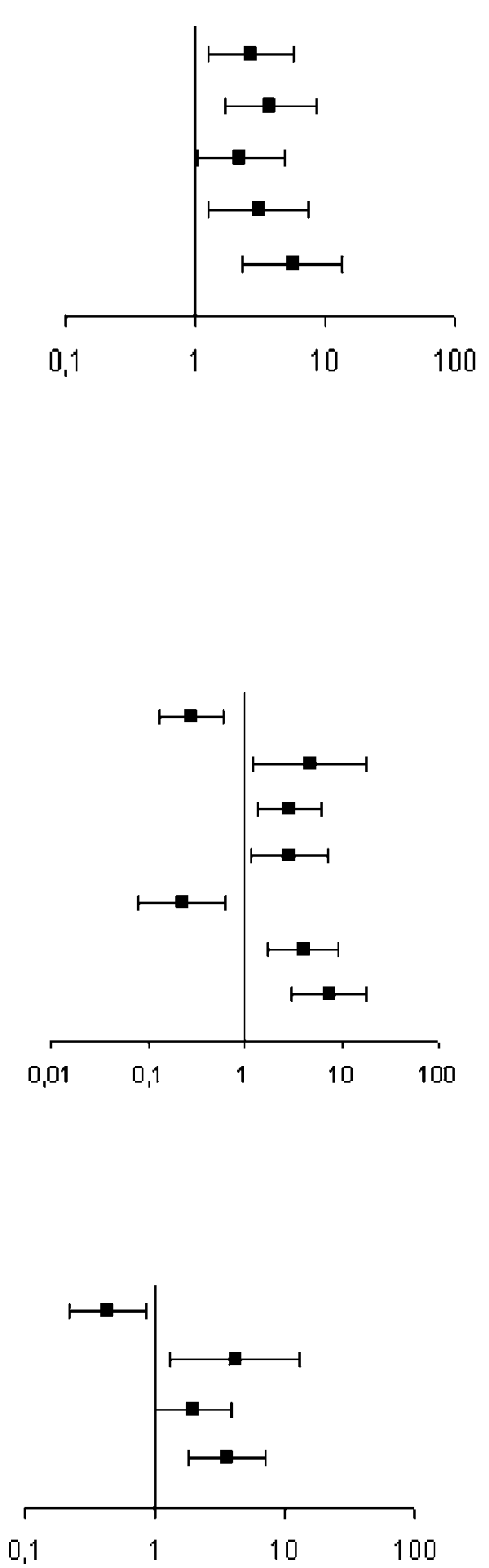

Figure 1 Continued.

adult families, did not remain its significance as a risk factor for separation anxiety. Mother with non-university education changed from being a risk factor for Separation anxiety to become a protective factor for General anxiety. The CBCL score prior to hospitalization in this population has been shown to have an impact on the PHBQ total score (8). When adding this information in multivariate logistic model, the result was that high CBCL scores was a risk fac-

\section{Eating disturbances}

Domicile in urban area

Tonsillectomy

Pain in recovery room, moderate to

severe

Nausea at home

Worst pain at home moderate to severe

\section{Regression Aggression}

Age more than 5 years

Only one adult in family

CBCL group high

Tonsillectomy vs. Other procedure

Midazolam in premedication

Postoperative nausea

Worst pain at home moderate to severe

\section{Sleep anxiety}

Age more than 5 years

Only one adult in family

$\mathrm{CBCL}$ group high

Worst pain at home moderate to severe

tor for Regression-aggression and Sleep anxiety. The finding that children with two or more siblings is more at risk in the Separation anxiety subscale, is somewhat consistent with the finding in a recent published study (11), although in this, the increased problematic behaviour was related to children with two or more older siblings. Contradictory to this, Kain at al. found that children with no siblings were more at risk in the separation anxiety subscale (9). 
The impact on children with earlier experience from hospitalization and anaesthesias on their behaviour was different and only on General anxiety-withdrawal subscale. Prior anaesthesias seem to have a protective effect, while two or more previous hospitalizations was a risk factor. Findings that previous difficult anaesthesia experiences (11) or healthcare (10) had negative impact on behaviour has been reported, but not that previous anaesthesia had beneficial role, even though that it may have a protective effect on behaviour in the immediate postoperative phase (35). In a recent study of two groups of frequently hospitalized children, one of them with frequent anaesthesias, there was no difference in their behaviour, which supported the hypothesis that chronic illness with hospitalizations rather than frequent anaesthesias was the main factor for their increased behavioural problems, measured with the CBCL (36).

The temperament of the child may have influence on later behaviour changes $(9,37)$, the present study shows that having more behavioural problems before hospitalization, assessed by CBCL, seems to be a risk to worsened postoperative behavioural problems in the Sleep anxiety and Regression-aggression subscales. This has also been observed by Lumley at al. (38)

\section{Hospital associated factors}

Day surgery versus inpatient care has been widely studied, and the general finding, as in the present study $(3,11,30)$, was that day surgery had less negative influence on behaviour. Staying two nights or more in hospital was found to be a risk factor also found by Stargatt et al. (11). A published meta analysis (3) found a tendency that stay in hospital 2-3 days had worse outcome than staying even longer. The only procedure which was tested in the present model was tonsillectomy, and the reason to this was that this procedure had more and longer duration of pain than other procedures and had been investigated in other studies. Tonsillectomy was found to have a negative impact on behaviour, this has not been found in other studies $(3,11,13)$.

\section{Anaesthesia induction}

Premedication with midazolam seems to have a protective effect which is in agreement with findings in previous studies $(19,20)$, although one study has shown negative effects (21) and others have found no influence (11). In the present study, premedication was not given in a randomized way, and no firm conclusions can be made based on this observation. Anaesthesia induction technique did not show any significant influence on behaviour. This is consistent with other studies, although there has been trends towards less negative impact of intravenous/rectal induction compared to inhalational induction $(14,24)$.

\section{Child and parental anxiety}

Child anxiety at anaesthesia induction seems to be a predictor to later problematic behaviour independent of midazolam premedication. An association between child anxiety and later behaviour problems has been previously shown in some studies $(9,12,13)$ but not all $(11)$. Parental anxiety has been shown to have influence on later behaviour $(9,11,12)$. Kain et al. state that preoperative anxiety and postoperative distress are linked together (12), but the present study shows that they independently predict increased negative behavioural outcome. Preparation programs have been extensively used and evaluated with the PHBQ with different results $(1,11,18,28-30)$. In this study only standard written information was sent to the family in advance. The anaesthesiologist made a routine visit to the child and parent at hospital. The simple evaluation with a VAS showed that very few felt less adequately prepared and there was no relation to the subsequent behaviour scoring.

\section{Pain}

Pain as a risk factor to increased PHBQ score has been studied by several groups and the results are contradictory; Kain (9), Vernon (4) and Stargatt (11) could not find any connection while Kotiniemi (10) found one. Our present study shows a strong correlation between pain and problematic behaviour. One possible explanation for these seemingly contradictory results is that there appears to be variation in pain reporting based on when in the course of the hospitalization the pain is assessed. Those who found no correlation measured only pain at hospital, but the present study, as well as Kotiniemi's, studied pain both at hospital and at home. Even though pain in the recovery room seems to have some influence, the major association is between pain at home and behaviour. Behaviour as an predictor pain is used in Parents Postoperative Pain Measure instrument (39). This instrument has items related to sleep, eating and separation anxiety like the PHBQ. It is unclear why postoperative pain in the hospital has demonstrated little or no impact on later behaviour, while pain at home has. Parents may have difficulty detecting theirs child's pain and they learned this by trial and error (40). Parents may have several misconceptions about children's pain, e.g. having higher pain thresholds, that children would not remember the pain or worry about addiction to analgesics $(40,41)$. Even if some discharge instructions are given, the parent may feel overwhelmed and worried of their child's postoperative recovery at home (42).

\section{Nausea}

Nausea as a predictor to behaviour changes has not been reported, as far we know. There have been investigations concerning preoperative anxiety as an predictor of nausea, but no correlation was found (43). In this study, primary nausea and vomiting in the hospital had impact on subsequent problematic behaviour, and nausea at home had impact on eating disturbances.

\section{Methodological considerations}

First, the drop frequency is quite high; the response rate was $55 \%$. Seventy-one questionnaires were lost due to administrative reasons. If these were not included in the analysis, and this potentially could be motivated since 
administrative loss of results was presumably a random process which would not introduce bias, then the response rate would have been $63 \%$. There were also missing data from that collected at the hospital. Secondly, many studies are designed to measure a single intervention, e.g. premedication or induction technique. In this study, the anaesthesia was up to the discretion of the anaesthesiologist. It is possible that there were undetected confounders, e.g. additional premedications which were given to those children that may have been considered to be extra anxious.

PHBQ is used in different ways and exists in different versions which make some results using this tool difficult to compare. Most study groups used the 27-item version, which was originally presented by Vernon et al. (4), but in their validation they noted that four items were deleted after performing factor analyse. The Finnish groups (Kotiniemi $(10,24,27)$, Tuomilehto (33)) used an abbreviated version which, to our knowledge, is not validated. The cut-off value for determination of problematic behaviour is also different. Thirdly, the influence of cultural factors may limit the ability to make indepth comparison between studies performed in different nations.

\section{CONCLUSIONS}

About a third of the children who visit the hospital and are subjected to a diagnostic or surgical procedure including anaesthesia will develop some negative behaviour when returning home. Moderate-to-severe postoperative pain at home is a strong risk factor, but less at hospital. Other risk factors include anxiety/distress at anaesthesia induction and in the recovery room. Nausea especially in the postoperative period in hospital is another risk factor. Some of those risk factors are avoidable, and this includes improving treatment of pain at home, instructions to parents and providing them with adequate analgesics (42), and the prophylactic use of anti-emetics in risk groups. Information of behavioural changes and parental participating in their children's hospital care has been shown to reduce posthospital problematic behavioural change (44).

Extra attention may be warranted for children who are at greater risk for later behavioural problems, including those from single parent families, younger children, those who stays at hospital for a longer time, have previous behavioural problems and has been hospitalized several times. Steps could be taken to make the anaesthesia induction as much as possible a non-stressful event. Living in a rural area, previous anaesthesias and midazolam in premedication seems to have a protective effect.

\section{ACKNOWLEDGEMENTS}

This study was supported by a grant from Oscarsfonden and Insamlingsfonden from the Umeå University. The authors thank Karin Lundbäck and Minette Wilén for their work with data collection, Arvid Otterlind for his work with data collection in Drottning Silvia's Child and Adolscent's Hospital Gothenburg and Michael Haney for his help with the English language.

\section{References}

1. Visintainer MA, Wolfer JA. Psychological preparation for surgery pediatric patients: the effects on children's and parents' stress responses and adjustment. Pediatrics 1975 Aug; 56: 187-202.

2. Zetterstrom R. Responses of children to hospitalization. Acta Paediatr Scand 1984 May; 73: 289-95.

3. Thompson RH, Vernon DT. Research on children's behavior after hospitalization: a review and synthesis. J Dev Behav Pediatr 1993 Feb; 14: 28-35.

4. Vernon DT, Schulman JL, Foley JM. Changes in children's behavior after hospitalization. Some dimensions of response and their correlates. Am J Dis Child 1966 Jun; 111: 581-93.

5. Karling M SH, Hägglöf B. Behavioural Changes after Anaesthesia: Validity and liability of the Post Hospitalisation Behaviour Questionnaire in a Swedish Paediatric Population. Acta Paediatrica 2005; 95: 340-6.

6. Achenbach T. Integrative guide for the 1991 CBCL/4-18,YSR and TRF profiles. Burlington, VT: University of Vermont, Department of Psychiatry, 1991.

7. Achenbach T. Manual for the Child Behaviour Checklist/2-3 and 1992 Profile. Burlington, VT: University of Vermont, Department of Psychiatry, 1992.

8. Karling M HB. Child behaviour after anaesthesia: Association of socioeconomic factors and Child Behaviour Checklist to the Post Hospital Behaviour Questionnaire. Acta Paediatr Scand 2006; In press.

9. Kain ZN, Mayes LC, O'Connor TZ, Cicchetti DV. Preoperative anxiety in children. Predictors and outcomes. Arch Pediatr Adolesc Med 1996 Dec; 150: 1238-45.

10. Kotiniemi LH, Ryhanen PT, Moilanen IK. Behavioural changes in children following day-case surgery: a 4-week follow-up of 551 children. Anaesthesia 1997 Oct; 52: 970-6.

11. Stargatt R, Davidson AJ, Huang GH, Czarnecki C, Gibson MA, Stewart SA, et al. A cohort study of the incidence and risk factors for negative behavior changes in children after general anesthesia. Paediatr Anaesth 2006 Aug; 16: 846-59.

12. Kain ZN, Caldwell-Andrews AA, Maranets I, McClain B, Gaal $\mathrm{D}$, Mayes LC, et al. Preoperative anxiety and emergence delirium and postoperative maladaptive behaviors. Anesth Analg 2004 Dec; 99: 1648-54, table of contents.

13. Kain ZN, Wang SM, Mayes LC, Caramico LA, Hofstadter MB Distress during the induction of anesthesia and postoperative behavioral outcomes. Anesth Analg 1999 May; 88: 1042-7.

14. Aguilera IM, Patel D, Meakin GH, Masterson J. Perioperative anxiety and postoperative behavioural disturbances in children undergoing intravenous or inhalation induction of anaesthesia. Paediatr Anaesth 2003 Jul; 13: 501-7.

15. Kain ZN, Mayes LC, Caramico LA, Silver D, Spieker M, Nygren MM, et al. Parental presence during induction of anesthesia. A randomized controlled trial. Anesthesiology 1996 May; 84: 1060-7.

16. Kain ZN, Mayes LC, Wang SM, Caramico LA, Hofstadter MB. Parental presence during induction of anesthesia versus sedative premedication: which intervention is more effective? Anesthesiology 1998 Nov; 89: 1147-56; discussion 9A-10A.

17. Elander G, Nilsson A, Lindberg T. Behavior in four-year-olds who have experienced hospitalization and day care. Am J Orthopsychiatry 1986 Oct; 56: 612-6.

18. Watson A, Visram A. The developing role of play preparation in paediatric anaesthesia. Paediatr Anaesth 2000; 10: 685-6.

19. Payne KA, Coetzee AR, Mattheyse FJ, Heydenrych JJ. Behavioural changes in children following minor surgery-is premedication beneficial? Acta Anaesthesiol Belg 1992; 43: $173-9$. 
20. Kain ZN, Mayes LC, Wang SM, Hofstadter MB. Postoperative behavioral outcomes in children: effects of sedative premedication. Anesthesiology 1999 Mar; 90: 758-65.

21. McGraw T, Kendrick A. Oral midazolam premedication and postoperative behaviour in children. Paediatr Anaesth 1998; 8: 117-21.

22. Padfield NL, Twohig MM, Fraser AC. Temazepam and trimeprazine compared with placebo as premedication in children. An investigation extended into the first 2 weeks at home. Br J Anaesth 1986 May; 58: 487-93.

23. Meyers EF, Muravchick S. Anesthesia induction technics in pediatric patients: a controlled study of behavioral consequences. Anesth Analg 1977 Jul-Aug; 56: 538-42.

24. Kotiniemi LH, Ryhanen PT. Behavioural changes and children's memories after intravenous, inhalation and rectal induction of anaesthesia. Paediatr Anaesth 1996; 6: 201-7.

25. Scaife JM, Campbell I. A comparison of the outcome of day-care and inpatient treatment of paediatric surgical cases. $J$ Child Psychol Psychiatry 1988 Mar; 29: 185-98.

26. Steward DJ. Experiences with an outpatient anesthesia service for children. Anesth Analg 1973 Nov-Dec; 52: 877-80.

27. Kotiniemi LH, Ryhanen PT, Moilanen IK. Behavioural changes following routine ENT operations in two-to-tenyear-old children. Paediatr Anaesth 1996; 6: 45-9.

28. Margolis JO, Ginsberg B, Dear GL, Ross AK, Goral JE, Bailey AG. Paediatric preoperative teaching: effects at induction and postoperatively. Paediatr Anaesth 1998; 8: 17-23.

29. Wolfer JA, Visintainer MA. Prehospital psychological preparation for tonsillectomy patients: effects on children's and parents' adjustment. Pediatrics 1979 Nov; 64: 646-55.

30. Vernon DT, Thompson RH. Research on the effect of experimental interventions on children's behavior after hospitalization: a review and synthesis. J Dev Behav Pediatr 1993 Feb; 14: 36-44.

31. Caldas JCS, Pais-Ribeiro JL, Carneiro SR. General anesthesia, surgery and hospitalization in children and their effects upon cognitive, academic, emotional and sociobehavioral development - a review. Pediatric Anesthesia 2004; 14: 910-5.

32. Watson AT, Visram A. Children's preoperative anxiety and postoperative behaviour. Paediatr Anaesth 2003 Mar; 13: 188-204.

33. Tuomilehto H, Kokki H, Ahonen R, Nuutinen J. Postoperative behavioral changes in children after adenoidectomy. Arch Otolaryngol Head Neck Surg 2002 Oct; 128: 1159-64.

34. Bosenberg A, Thomas J, Lopez T, Kokinsky E, Larsson LE. Validation of a six-graded faces scale for evaluation of postoperative pain in children. Paediatr Anaesth 2003 Oct; 13: 708-13.

35. Caumo W, Broenstrub JC, Fialho L, Petry SM, Brathwait O, Bandeira D, et al. Risk factors for postoperative anxiety in children. Acta Anaesthesiol Scand 2000 Aug; 44: 782-9.

36. Kayaalp L, Bozkurt P, Odabasi G, Dogangun B, Cavusoglu P, Bolat N, et al. Psychological effects of repeated general anesthesia in children. Paediatr Anaesth 2006 Aug; 16: 822-7.

37. McClowry SG. The relationship of temperament to pre- and posthospitalization behavioral responses of school-age children. Nurs Res 1990 Jan-Feb; 39: 30-5.

38. Lumley MA, Melamed BG, Abeles LA. Predicting children's presurgical anxiety and subsequent behavior changes. J Pediatr Psychol 1993 Aug; 18: 481-97.

39. Finley GA, Chambers CT, McGrath PJ, Walsh TM. Construct validity of the parents' postoperative pain measure. Clin J Pain 2003 Sep-Oct; 19: 329-34.

40. Gedaly-Duff V, Ziebarth D. Mothers' management of adenoid-tonsillectomy pain in 4- to 8-year-olds: a preliminary study. Pain 1994 Jun; 57: 293-9.

41. Kankkunen PM, Vehvilainen-Julkunen KM, Pietila AM. Children's postoperative pain at home: family interview study. Int J Nurs Pract 2002 Feb; 8: 32-41.

42. Bastable A, Rushforth H. Parents' management of their child's postoperative pain. Paediatr Nurs 2005 Dec; 17: 14-7.

43. Wang SM, Kain ZN. Preoperative anxiety and postoperative nausea and vomiting in children: is there an association? Anesth Analg 2000 Mar; 90: 571-5.

44. Melnyk BM, Feinstein NF. Mediating functions of maternal anxiety and participation in care on young children's posthospital adjustment. Res Nurs Health 2001 Feb; 24: $18-26$.

\section{Supplementary material}

The following supplementary material is available for this article:

Table S1 Demographic and prehospital data

Table S2 Preoperative hospital data

Table S3 Postoperative hospital data

Table S4 Postoperative home data

Table S5 Type of surgery and length of stay

Figure S1 Flow sheet, number of patients, dropouts and data collected.

Figure S2 Number of children with problematic outcome (PHBQ score $>75$ ) by the total PHBQ score.wn.

This material is available as part of the online article from: http://www.blackwell-synergy.com/doi/abs/10.1111/j.16512227.2007.00258.x

(This link will take you to the article abstract).

Please note: Blackwell Publishing is not responsible for the content or functionality of any supplementary materials supplied by the authors. Any queries (other than missing material) should be directed to the corresponding author for the article. 